\title{
A Globally Convergent Parallel SSLE Algorithm for Inequality Constrained Optimization
}

\author{
Zhijun Luo ${ }^{1}$ and Lirong Wang ${ }^{2}$ \\ ${ }^{1}$ The Department of Mathematics and Econometrics, Hunan University of Humanities, Science and Technology, Loudi 417000, China \\ ${ }^{2}$ The Department of Information Science and Engineering, Hunan University of Humanities, Science and Technology, \\ Loudi 417000, China
}

Correspondence should be addressed to Zhijun Luo; ldlzj123@163.com

Received 31 January 2014; Accepted 2 March 2014; Published 3 April 2014

Academic Editor: Cedric Yiu

Copyright (c) 2014 Z. Luo and L. Wang. This is an open access article distributed under the Creative Commons Attribution License, which permits unrestricted use, distribution, and reproduction in any medium, provided the original work is properly cited.

\begin{abstract}
A new parallel variable distribution algorithm based on interior point SSLE algorithm is proposed for solving inequality constrained optimization problems under the condition that the constraints are block-separable by the technology of sequential system of linear equation. Each iteration of this algorithm only needs to solve three systems of linear equations with the same coefficient matrix to obtain the descent direction. Furthermore, under certain conditions, the global convergence is achieved.
\end{abstract}

\section{Introduction}

Consider the following inequality constrained optimization problems:

$$
\begin{array}{ll}
\min & f(x) \\
\text { s.t. } & g_{j}(x) \leq 0, \quad j \in I=\{1, \ldots, m\},
\end{array}
$$

where $f(x): R^{n} \rightarrow R, g(x): R^{n} \rightarrow R$ are continuously differentiable. We denote

$$
\begin{gathered}
X=\left\{x \in R^{n} \mid g_{j}(x) \leq 0, j \in I\right\}, \\
X^{0}=\left\{x \in R^{n} \mid g_{j}(x)<0, j \in I\right\}, \\
I(x)=\left\{j \mid g_{j}(x)=0, j \in I\right\} .
\end{gathered}
$$

To solve the problem (1), there are two type methods with superlinear convergence: sequential quadratic programming (SQP) type algorithms (see [1-4], etc.) and SSLE (sequential system of linear equations) type algorithms (see [5-9], etc.). In general, since SQP algorithms are necessary to solve one or more quadratic programming subproblems in single iteration, the computation effort is very large.
SSLE algorithms were proposed to solve the problem (1), in which an iteration similar to the following linear system was considered:

$$
\begin{gathered}
H d_{0}+\nabla_{x} L\left(x, \lambda_{0}\right)=0, \\
\mu_{j} \nabla g_{j}(x)^{T} d_{0}+\lambda_{0 j} g_{j}(x)=0, \quad j=1 \sim m,
\end{gathered}
$$

where $L(x, \lambda)=f(x)+\sum_{j=1}^{m} \lambda_{j} g_{j}(x)$ is Lagrangian function, $H$ is an estimate of the Hessian of $L, x$ is the current estimate of a solution $x^{*}, d_{0}$ is the search direction, and $\lambda_{0}$ is the next estimate of the Kuhn-Tucker multiplier vector associated with $x^{*}$. Obviously, it is simpler to solve system of linear equations than to solve the QP (quadratic programming) problem with inequality constraints.

In addition, parallel variable distribution (PVD) algorithm [10] is a method that distributes the variables among parallel processors. The problem is parted into many respective subproblems and each subproblem is arranged to a different processor in it. Each processor has the primary responsibility for updating its block of variables while allowing the remaining secondary variables to change in a restricted fashion along some easily computable directions. In 2002, Sagastizábal and Solodov [11] proposed two new variants of PVD for the constrained case. Without assuming convexity 
of constraints, but assuming block-separable structure, they showed that PVD subproblems can be solved inexactly by solving their quadratic programming approximations. Han et al. [12] proposed an asynchronous PVT algorithm for solving large-scale linearly constrained convex minimization problems with the idea in 2009, which is based on the idea that a constrained optimization problem is equivalent to a differentiable unconstrained optimization problem by introducing the Fischer function. In 2011, Zheng et al. [13] gave a parallel SSLE algorithm, in which the PVD subproblems are solved inexactly by serial sequential linear equations, for solving large-scale constrained optimization with block-separable structure. Without assuming the convexity of constraints, the algorithm is proved to be globally convergent to a KKT point.

In this paper, we use Zhu [8] as our main reference on SSLE-type PVD method for problem (1). Suppose that the problem (1) has the following block-separable structure:

$$
\begin{gathered}
x=\left(x_{1}, x_{2}, \ldots, x_{p}\right), \quad x_{l} \in R^{n_{l}}, l=1,2, \ldots, p, \\
\sum_{l=1}^{p} n_{l}=n, \\
g(x)=\left(g_{1}\left(x_{1}\right), g_{2}\left(x_{2}\right), \ldots, g_{p}\left(x_{p}\right)\right), \\
g_{l}\left(x_{l}\right): R^{n_{l}} \longrightarrow R^{m_{l}}, \quad \sum_{l=1}^{p} m_{l}=m, \\
I=\left(I_{1}, I_{2}, \ldots, I_{p}\right)=\{1,2, \ldots, m\}, \\
I_{l}\left(x_{l}\right)=\left\{l_{j} \mid g_{l j}\left(x_{l}\right)=0, l_{j} \in I_{l}=\left\{1,2, \ldots, m_{l}\right\}\right\} .
\end{gathered}
$$

Then, the problem is distributed into $p$ parallel subproblems which have been computed by the $p$ parallel processors. In the algorithm, at each iteration, the search direction is obtained by solving three systems of linear equations with the same coefficient matrix, which guarantees that every iteration is feasible. Thereby, the computational effort of the proposed algorithm is reduced further. Furthermore, its global convergence is obtained under some suitable conditions.

The remaining part of this paper is organized as follows. In Section 2, a parallel SSLE algorithm is presented. Global convergence is established under some basic assumptions in Section 3. And concluding remarks are given in the last section.

\section{Description of Algorithm}

Now we state our algorithm as follows.

\section{Algorithm}

Step 0 (initialization). Given a starting point $x^{0} \in X^{0}$. Choose parameters $\theta, \sigma \in(0,1), \alpha \in(0,1 / 2), 2<\tau<\delta<3, \bar{\mu}>0$, $0<\mu_{l_{j}}^{0} \leq \bar{\mu}$, and $\left(l_{j} \in I_{l}\right)$ and an initial symmetric positive definite matrix $H_{l}^{0} \in R^{n_{l} \times n_{l}}$. Set $k=0$.

Step 1 (parallelization). For each processor $l \in\{1,2, \ldots, p\}$, let

$$
\begin{gathered}
N_{l}^{k}=\left(\nabla g_{l_{j}}\left(x_{l}^{k}\right), l_{j} \in I_{l}\right), \\
G_{l}^{k}=\operatorname{diag}\left(g_{l_{j}}\left(x_{l}^{k}\right), l_{j} \in I_{l}\right), \\
M_{l}^{k}=\operatorname{diag}\left(\mu_{l_{j}}^{k}, l_{j} \in I_{l}\right) .
\end{gathered}
$$

1.1 Computation of the Newton Direction. Solve the following system of linear equations:

$$
\left(\begin{array}{cc}
H_{l}^{k} & N_{l}^{k} \\
M_{l}^{k}\left(N_{l}^{k}\right)^{T} & G_{l}^{k}
\end{array}\right)\left(\begin{array}{l}
d \\
\lambda
\end{array}\right)=-\left(\begin{array}{c}
\nabla_{l} f\left(x_{l}^{k}\right) \\
0
\end{array}\right) .
$$

Let $\left(d_{l}^{0 k}, \pi_{l}^{k}\right)$ be the solution. If $d_{0}^{k}=0$, stop.

1.2 Computation of the Descent Direction. Solve the following system of linear equations:

$$
\left(\begin{array}{cc}
H_{l}^{k} & N_{l}^{k} \\
M_{l}^{k}\left(N_{l}^{k}\right)^{T} & G_{l}^{k}
\end{array}\right)\left(\begin{array}{l}
d \\
\lambda
\end{array}\right)=-\left(\begin{array}{c}
\nabla_{l} f\left(x_{l}^{k}\right) \\
\left\|d_{l}^{0 k}\right\|^{\delta} e_{l}
\end{array}\right)
$$

where $e_{l}=(1, \ldots, 1)^{T} \in R^{m_{l}}$. Let $\left(d_{l}^{1 k}, \tilde{\pi}_{l}^{k}\right)$ be the solution.

1.3 Computation of the Main Search Direction. Establish a convex combination of $d_{l}^{0 k}$ and $d_{l}^{1 k}$ :

$$
\begin{aligned}
d_{l}^{k} & =\left(1-\beta_{l}^{k}\right) d_{l}^{0 k}+\beta_{l}^{k} d_{l}^{1 k}, \\
\lambda_{l}^{k} & =\left(1-\beta_{l}^{k}\right) \pi_{l}^{k}+\beta_{l}^{k} \tilde{\pi}_{l}^{k},
\end{aligned}
$$

where

$$
\begin{aligned}
\beta_{l}^{k}=\max \left\{\beta \in(0,1] \mid(1-\beta) \nabla_{l} f\left(x_{l}^{k}\right)^{T} d_{l}^{0 k}\right. \\
\left.+\beta \nabla_{l} f\left(x_{l}^{k}\right)^{T} d_{l}^{1 k} \leq \theta \nabla_{l} f\left(x_{l}^{k}\right)^{T} d_{l}^{0 k}\right\} .
\end{aligned}
$$

1.4 Computation of the High-Order Corrected Direction. Set

$$
L_{l}^{k}=\left\{l_{j} \in I_{l} \mid g_{l_{j}}\left(x_{l}^{k}\right) \geq-\lambda_{l_{j}}^{k}\right\} .
$$

Solve the following system of linear equations:

$$
\left(\begin{array}{cc}
H_{l}^{k} & N_{l}^{k} \\
M_{l}^{k}\left(N_{l}^{k}\right)^{T} & G_{l}^{k}
\end{array}\right)\left(\begin{array}{l}
d \\
\lambda
\end{array}\right)=-\left(\begin{array}{c}
\nabla_{l} f\left(x_{l}^{k}\right) \\
\left\|d_{l}^{0 k}\right\|^{\tau} e_{l}+M_{l}^{k} \tilde{g}_{l}^{k}
\end{array}\right),
$$

where

$$
\begin{gathered}
\tilde{g}_{l}^{k}=\left(\tilde{g}_{l_{j}}^{k}, l_{j} \in I_{l}\right), \\
\tilde{g}_{l_{j}}^{k}= \begin{cases}g_{l_{j}}\left(x_{l}^{k}+d_{l}^{k}\right), & l_{j} \in L_{l}^{k}, \\
0, & l_{j} \in I_{l} \backslash L_{l}^{k} .\end{cases}
\end{gathered}
$$


Let $\left(\tilde{d}_{l}^{k}+d_{l}^{k}, \tilde{\lambda}_{l}^{k}+\lambda_{l}^{k}\right)$ be the solution. If $\left\|\tilde{d}_{l}^{k}\right\|>\left\|d_{l}^{k}\right\|$, set $\tilde{d}_{l}^{k}=0$.

Step 2 (synchronization). Let

$$
\begin{gathered}
d^{k}=\left(d_{1}^{k}, d_{2}^{k}, \ldots, d_{p}^{k}\right), \quad \tilde{d}^{k}=\left(\tilde{d}_{1}^{k}, \tilde{d}_{2}^{k}, \ldots, \tilde{d}_{p}^{k}\right), \\
\lambda^{k}=\left(\lambda_{1}^{k}, \lambda_{2}^{k}, \ldots, \lambda_{p}^{k}\right), \quad J_{k}=\left\{j \in I \mid g_{j}\left(x^{k}\right) \geq \lambda_{j}^{k}\right\} .
\end{gathered}
$$

2.1 The Line Search. Compute $t_{k}$, the first number $t$ in the sequence $\{1,1 / 2,1 / 4, \ldots\}$ satisfying

$$
\begin{gathered}
f\left(x^{k}+t d^{k}+t^{2} \tilde{d}^{k}\right) \leq f\left(x^{k}\right)+\alpha t \nabla f\left(x^{k}\right)^{T} d^{k}, \\
g_{j}\left(x^{k}+t d^{k}+t^{2} \tilde{d}^{k}\right) \leq g_{j}\left(x^{k}\right), \quad j \in J_{k}, \\
g_{j}\left(x^{k}+t d^{k}+t^{2} \widetilde{d}^{k}\right)<0, \quad j \in I \backslash J_{k} .
\end{gathered}
$$

Step 3 (update). Obtain $H_{l}^{k+1}$ by updating the positive definite matrix $H_{l}^{k}$ using some quasi-Newton formulas. Set

$$
\mu_{l_{j}}^{k+1}=\min \left\{\max \left\{\pi_{l_{j}}^{k},\left\|d_{l}^{0 k}\right\|\right\}, \bar{\mu}\right\}, \quad l_{j} \in I_{l},
$$

and $x^{k+1}=x^{k}+t_{k} d^{k}+t_{k}^{2} \tilde{d}^{k}$. Let $k=k+1$. Go back to Step 1 .

\section{Global Convergence of Algorithm}

We make the following general assumptions and let them hold throughout the paper.

(H3.1) For $l=1,2, \ldots, p$, the sets $X_{l}=\left\{x_{l} \mid g_{l}\left(x_{l}\right) \leq 0\right\}$ and $X_{l}^{0}=\left\{x_{l} \mid g_{l}\left(x_{l}\right)<0\right\}$ are nonempty. The set $X_{l} \cap\left\{x_{l} \mid f\left(x_{l}\right) \leq f\left(x_{l}^{0}\right)\right\}$ is compact.

(H3.2) The functions $f, g_{j}$, and $j \in I$ are continuously differentiable.

(H3.3) For all $x_{l} \in X_{l}$, the set of vectors $\left\{\nabla g_{l_{j}}\left(x_{l}\right): l_{j} \in I_{l}\left(x_{l}\right)\right\}$ is linearly independent.

(H3.4) There exist $a, b>0$, such that $a\|d\|^{2} \leq d^{T} H_{l}^{k} d \leq$ $b\|d\|^{2}$, for all $k \in R$ and $d \in R^{n_{l}}$.

Lemma 1. For any $x_{l} \in X_{l}$, any positive-definite matrix $H_{l} \in$ $R^{n_{l} \times n_{l}}$, and nonnegative vector $\mu_{l}=\left(\mu_{l_{j}}, l_{j} \in I_{l}\right) \in R^{m_{l}}$ such that $\mu_{l j}>0, l_{j} \in I_{l}\left(x_{l}\right)$, the matrix

$$
F\left(x_{l}, H_{l}, \mu_{l}\right)=\left(\begin{array}{cc}
H_{l} & N_{l} \\
M_{l} N_{l}^{T} & G_{l}
\end{array}\right)
$$

is nonsingular, where $N_{l}=\left(\nabla g_{l j}\left(x_{l}\right), l_{j} \in I_{l}\right) \in R^{n_{l} \times m_{l}}, M_{l}=$ $\operatorname{diag}\left(\mu_{l j}, l_{j} \in I_{l}\right) \in R^{m_{l} \times m_{l}}$, and $G_{l}=\operatorname{diag}\left(g_{l j}\left(x_{l}\right), l_{j} \in I_{l}\right) \in$ $R^{m_{l} \times m_{l}}$.
Proof. We need only to prove that $\left(d_{l}, \lambda_{l}\right)=(0,0) \in R^{n_{l} \times m_{l}}$ is the unique solution of the following linear equations:

$$
F\left(x_{l}, H_{l}, \mu_{l}\right)\left(\begin{array}{l}
d_{l} \\
\lambda_{l}
\end{array}\right)=0, \quad \text { that is, }\left\{\begin{array}{l}
H_{l} d_{l}+N_{l} \lambda_{l}=0, \\
M_{l} N_{l}^{T} d_{l}+G_{l} \lambda_{l}=0 .
\end{array}\right.
$$

Now consider the cases $l_{j} \in I_{l}\left(x_{l}\right)$ and $l_{j} \notin I_{l}\left(x_{l}\right)$ separately.

For $l_{j} \in I_{l}\left(x_{l}\right)$, if $g_{l j}\left(x_{l}\right)=0$ and $\mu_{l j}>0$, it follows from (19) that

$$
\nabla g_{l j}\left(x_{l}\right)^{T} d_{l}=0
$$

By (19), we have

$$
\sum_{l_{j} \in I_{l}\left(x_{l}\right)} \lambda_{l j} \nabla g_{l j}\left(x_{l}\right)=0
$$

Then from the assumption (H3.3), it shows that $\lambda_{l_{j}}=0\left(\forall l_{j} \in\right.$ $\left.I_{l}\left(x_{l}\right)\right)$.

For $l_{j} \notin I_{l}\left(x_{l}\right)$, if $\mu_{l j}=0$, it follows from the first equation of (19) that

$$
\lambda_{l_{j}}=0, \quad d_{l_{j}}=0 .
$$

If $\mu_{l j}>0$, it follows from the second equation of (19) that

$$
\nabla g_{l j}\left(x_{l}\right)^{T} d_{l}=-\frac{\lambda_{l_{j}}}{\mu_{l j}} g_{l j}\left(x_{l}\right) .
$$

Hence, if $\mu_{l j} \geq 0$, combine (22) and (23), and from the first equation of (19) and the assumption (H3.4), we get

$$
\begin{aligned}
0 & \leq d_{l}^{T} H_{l} d_{l}=-\lambda_{l}^{T} N_{l}^{T} d_{l} \\
& =-\sum_{l_{j} \notin I_{l}\left(x_{l}\right), \mu_{l j}>0} \lambda_{l j}\left(\nabla g_{l j}\left(x_{l}\right)^{T} d_{l}\right) \\
& =\sum_{l_{j} \notin I_{l}\left(x_{l}\right), \mu_{l j}>0} \frac{\left(\lambda_{l j}\right)^{2}}{\mu_{l j}} g_{l j}\left(x_{l}\right) \leq 0 .
\end{aligned}
$$

It shows that $d_{l_{j}}=0$ and $\lambda_{l_{j}}=0\left(\forall l_{j} \notin I_{l}\left(x_{l}\right)\right)$.

Lemma 2. For $l=1,2, \ldots, p$,

(1) if $d_{l}^{0 k}=0$, then $x^{k}$ is a KKT point of (1);

(2) if $d_{l}^{0 k} \neq 0$, then $d_{l}^{k}$ computed according to (8) is well defined and

$$
\begin{gathered}
\nabla_{l} f\left(x_{l}^{k}\right)^{T} d_{l}^{0 k} \leq-\left(d_{l}^{0 k}\right)^{T} H_{l}^{k} d_{l}^{0 k}<0, \\
\nabla_{l} f\left(x_{l}^{k}\right)^{T} d_{l}^{k} \leq \theta \nabla_{l} f\left(x_{l}^{k}\right)^{T} d_{l}^{0 k}<0,
\end{gathered}
$$

$$
\nabla g_{l_{j}}\left(x_{l}^{k}\right)^{T} d_{l}^{k}=-\frac{\lambda_{l_{j}}^{k}}{\mu_{l_{j}}^{k}} g_{l_{j}}\left(x_{l}^{k}\right)-\beta_{k}\left\|d_{l}^{0 k}\right\|^{\delta}, \quad l_{j} \in I_{l} \text {. }
$$


Proof. (1) It is obvious according to the definition of the KKT point of (1).

(2) If $d_{l}^{0 k} \neq 0$, from (6), we have

$$
\begin{aligned}
& \nabla_{l} f\left(x_{l}^{k}\right)^{T} d_{l}^{0 k}=-\left(d_{l}^{0 k}\right)^{T} H_{l}^{k} d_{l}^{0 k}-\left(N_{k} \pi^{k}\right)^{T} d_{l}^{0 k} \\
&=-\left(d_{l}^{0 k}\right)^{T} H_{l}^{k} d_{l}^{0 k}+\sum_{j \in I} \frac{\left(\pi_{l_{j}}^{k}\right)^{2}}{\mu_{l_{j}}^{k}} g_{l_{j}}\left(x^{k}\right) \\
& \leq-\left(d_{l}^{0 k}\right)^{T} H_{l}^{k} d_{l}^{0 k}<0, \\
& \nabla g_{l_{j}}\left(x^{k}\right)^{T} d_{l}^{0 k}=-\frac{\pi_{l_{j}}^{k}}{\mu_{l_{j}}^{k}} g_{l_{j}}\left(x^{k}\right), \quad l_{j} \in I_{l} .
\end{aligned}
$$

Thereby, from (9), there exists some $\beta \in(0,1]$, such that $\beta_{k}=$ $\beta \in(0,1]$; that is, $d_{l}^{k}$ is well defined. In addition, from (7), it follows that

$$
\nabla g_{l_{j}}\left(x_{l}^{k}\right)^{T} d_{1}^{k}=-\frac{\tilde{\pi}_{l_{j}}^{k}}{\mu_{l_{j}}^{k}} g_{l_{j}}\left(x_{l}^{k}\right)-\left\|d_{l}^{0 k}\right\|^{\delta}, \quad l_{j} \in I_{l} .
$$

Thus, from (8), it is clear to see that

$$
\begin{aligned}
\nabla g_{l_{j}}\left(x_{l}^{k}\right)^{T} d_{l}^{k} & =\left(1-\beta_{k}\right) \nabla g_{l_{j}}\left(x_{l}^{k}\right)^{T} d_{l}^{0 k}+\beta_{k} \nabla g_{j}\left(x_{l}^{k}\right)^{T} d_{l}^{1 k} \\
& =-\frac{\lambda_{l_{j}}^{k}}{\mu_{l_{j}}^{k}} g_{l_{j}}\left(x_{l}^{k}\right)-\beta_{k}\left\|d_{l}^{0 k}\right\|^{\delta}, \quad l_{j} \in I_{l}, \\
\nabla_{l} f\left(x_{l}^{k}\right)^{T} d_{l}^{k} & =\left(1-\beta_{k}\right) \nabla_{l} f\left(x_{l}^{k}\right)^{T} d_{l}^{0 k}+\beta_{k} \nabla_{l} f\left(x_{l}^{k}\right)^{T} d_{l}^{1 k} \\
& \leq \theta \nabla_{l} f\left(x_{l}^{k}\right)^{T} d_{l}^{0 k}<0 .
\end{aligned}
$$

The claim holds.

Lemma 3. The line search in Step 2 of the algorithm is well defined; that is, there exists $t_{k}>0$ such that (14)-(16) hold.

Proof. Firstly, for (14), since $f$ is continuously differentiable, we can see that

$$
\begin{aligned}
a_{k} & \triangleq f\left(x^{k}+t d^{k}+t^{2} \tilde{d}^{k}\right)-f\left(x^{k}\right)-\alpha t \nabla f\left(x^{k}\right)^{T} d^{k} \\
& =\nabla f\left(x^{k}\right)^{T}\left(t d^{k}+t^{2} \tilde{d}^{k}\right)-\alpha t \nabla f\left(x^{k}\right)^{T} d^{k}+o(t) \\
& =(1-\alpha) t \nabla f\left(x^{k}\right)^{T} d^{k}+o(t) .
\end{aligned}
$$

From (25), we have $\nabla_{l} f\left(x_{l}^{k}\right)^{T} d_{l}^{k}<0,(l \in\{1,2, \ldots, p\})$. Then we can obtain

$$
\nabla f\left(x^{k}\right)^{T} d^{k}=\sum_{l=1}^{p} \nabla_{l} f\left(x_{l}^{k}\right)^{T} d_{l}^{k}<0 .
$$

Thus, for $\alpha \in(0,1 / 2)$, there exists some $\bar{t}>0$, such that $a_{k} \leq$ $0, \forall t \in[0, \bar{t}]$.
On the other hand, from (25), if $l_{j} \in I_{l},(l \in\{1,2, \ldots, p\})$, we have

$$
\nabla g_{l_{j}}\left(x_{l}^{k}\right)^{T} d_{l}^{k}=-\frac{\lambda_{l_{j}}^{k}}{\mu_{l_{j}}^{k}} g_{l_{j}}\left(x_{l}^{k}\right)-\beta_{k}\left\|d_{l}^{0 k}\right\|^{\delta}, \quad l_{j} \in I_{l} .
$$

So, for all $j \in\{1,2, \ldots, m\}$,

$$
\begin{aligned}
\nabla g_{j}\left(x^{k}\right)^{T} d^{k} & =\sum_{l=1}^{p} \sum_{j=1}^{m_{l}} \nabla g_{l_{j}}\left(x_{l}^{k}\right)^{T} d_{l}^{k} \\
& =-\frac{\lambda_{j}^{k}}{\mu_{j}^{k}} g_{j}\left(x^{k}\right)-\beta_{k}\left\|d^{k 0}\right\|^{\delta}, \quad j \in I .
\end{aligned}
$$

Then, from (13), we obtain, for $j \in J_{k}$, that

$$
\begin{aligned}
b_{j}^{k} & \triangleq g_{j}\left(x^{k}+t d^{k}+t^{2} \tilde{d}^{k}\right)-g_{j}\left(x^{k}\right) \\
& =t \nabla g_{j}\left(x^{k}\right)^{T} d^{k}+o(t) \\
& =-\frac{\lambda_{j}^{k}}{\mu_{j}^{k}} t g_{j}\left(x^{k}\right)-t \beta_{k}\left\|d_{0}^{k}\right\|^{\delta}+o(t) \\
& \leq-\frac{1}{\mu_{j}^{k}} t g_{j}^{2}\left(x^{k}\right)-t \beta_{k}\left\|d_{0}^{k}\right\|^{\delta}+o(t) .
\end{aligned}
$$

So, there exists some $\bar{t}_{j}>0, j \in J_{k}$, such that $b_{j}^{k} \leq 0, \forall t \in$ $\left[0, \bar{t}_{j}\right]$; that is, (15) holds.

Thirdly, for (16), since $g$ is continuous and $g_{j}\left(x^{k}\right)<0$, there exists some $\bar{t}_{j}>0, j \notin J_{k}$, such that

$$
g_{j}\left(x^{k}+t d^{k}+t^{2} \widetilde{d}^{k}\right) \leq \frac{1}{2} g_{j}\left(x^{k}\right)<0 .
$$

Let $t_{k}=(1 / 2)^{i}=\min \left\{\bar{t}, \bar{t}_{j}, j \in I\right\}$; then the conclusion holds.

According to (H3.1), (H3.2), and (H3.4), we might assume that there exists a subsequence $K$ as well, such that

$$
\begin{array}{r}
x^{k} \longrightarrow x^{*}, \quad H_{l}^{k} \longrightarrow H_{l}^{*}, \quad d_{l}^{0 k} \longrightarrow d_{l}^{0 *}, \\
\pi_{l}^{k} \longrightarrow \pi_{l}^{*}, \quad \mu_{l}^{k} \longrightarrow \mu_{l}^{*}, \\
\\
k \in K
\end{array}
$$

In order to obtain the global convergence of the algorithm, we assume the following condition.

(H3.5) The number of stationary points of (1) is finite.

Theorem 4. The algorithm in Section 2 either stops at the KKT point $x^{k}$ of (1) in finite iteration or generates an infinite sequence $\left\{x^{k}\right\}$ whose all accumulation points are KKT points of (1). 
Proof. The first statement is obvious, the only stopping point being in Step 1.1. Firstly, we show that

$$
\left\{x^{k}\right\}_{k \in K} \longrightarrow x^{*}, \quad d^{0 k} \longrightarrow 0, \quad k \in K
$$

where $x^{*}=\left(x_{1}^{*}, x_{2}^{*}, \ldots, x_{p}^{*}\right), d^{0 k}=\left(d_{1}^{0 k}, d_{2}^{0 k}, \ldots, d_{p}^{0 k}\right)$.

Since $\left\{f\left(x^{k}\right)\right\}$ is monotonically decreasing, the facts $\left\{x^{k}\right\}_{k \in K} \rightarrow x^{*}$ and continuity of $f$ imply that

$$
f\left(x^{k}\right) \longrightarrow f\left(x^{*}\right), \quad k \longrightarrow \infty .
$$

For $l_{j} \in I_{l},(l \in\{1,2, \ldots, p\})$, suppose by contradiction that $d_{l}^{0 *} \neq 0$. Then, from (17), we have

$$
\mu_{l_{j}}^{k} \longrightarrow \mu_{l_{j}}^{*}>0, \quad l_{j} \in I_{l}, k \in K
$$

Hence, it is easy to prove that $\left(d_{l}^{0 *}, \pi_{l}^{*}\right)$ is the unique solution of the following linear system:

$$
\begin{gathered}
H_{l}^{*} d_{l}+\nabla_{l} f\left(x_{l}^{*}\right)+N_{l}^{*} \pi_{l}=0, \\
\mu_{l_{j}}^{*} \nabla g_{l_{j}}\left(x_{l}^{*}\right)^{T} d_{l}+\pi_{l_{j}} g_{l_{j}}\left(x_{l}^{*}\right)=0, \quad l_{j} \in I_{l},
\end{gathered}
$$

where $N_{l}^{*}=\left(\nabla g_{l_{j}}\left(x_{l}^{*}\right), l_{j} \in I_{l}\right)$. Then we can obtain

$$
\begin{gathered}
H^{*} d+\nabla f\left(x^{*}\right)+N^{*} \pi=0, \\
\mu_{j}^{*} \nabla g_{j}\left(x^{*}\right)^{T} d+\pi_{j} g_{j}\left(x^{*}\right)=0, \quad j \in I .
\end{gathered}
$$

Thereby,

$$
\nabla f\left(x^{*}\right)^{T} d^{0 *}<0 .
$$

Similar to (8), we define $d^{*}$, and by imitating the proof of Lemma 2, it follows that

$$
\begin{gathered}
\nabla f\left(x^{*}\right)^{T} d^{*}<0, \\
\nabla g_{j}\left(x^{*}\right)^{T} d^{*} \leq-\frac{\lambda_{j}^{*}}{\mu_{j}^{*}} g_{j}\left(x^{*}\right)-\beta^{*}\left\|d_{0}^{*}\right\|^{\delta}, \quad j \in I .
\end{gathered}
$$

From (41), (42), and the proof of Lemma 3, we can conclude that the step-size $t_{k}$ obtained by the linear search in Step 2.2 is bounded away from zero on $K$; that is,

$$
t_{k} \geq t_{*}=\inf \left\{t_{k}, k \in K\right\}>0, \quad k \in K .
$$

So, from (14), (37), and (42), we get

$$
\begin{aligned}
0 & =\lim _{k \in K}\left(f\left(x^{k+1}\right)-f\left(x^{k}\right)\right) \leq \lim _{k \in K} \alpha t_{k} \nabla f\left(x^{k}\right)^{T} d^{k} \\
& \leq \frac{1}{2} \alpha t_{*} \nabla f\left(x^{*}\right)^{T} d^{*}<0 .
\end{aligned}
$$

It is a contradiction, which shows that $d^{0 k} \rightarrow 0, k \in K$.

Furthermore, from (40), we have

$$
\nabla f\left(x^{*}\right)+N_{*} \pi^{*}=0, \quad \pi_{j}^{*} g_{j}\left(x^{*}\right)=0, \quad j \in I .
$$

If $g_{j}\left(x^{*}\right)<0, \forall j \in I$, then $\pi^{*}=0, \nabla f\left(x^{*}\right)=0$, and it is obvious that $x^{*}$ is a KKT point of (1).

Without loss of generality, we suppose that there exists some $j_{0} \in I$, such that $g_{j_{0}}\left(x^{*}\right)=0$. If $\pi_{j_{0}}^{*} \geq 0$, then it is easy to see that $x^{*}$ is a KKT point of (1). Suppose that $\pi_{j_{0}}^{*}<0$. Since there are only finitely many choices for sets $J_{k} \subseteq I$, we might assume, for $k \in K, k$ large enough, that $J_{k} \equiv J$ as well, where $J$ is a constant set. Obviously, $j_{0} \in J \neq \emptyset$. From condition (H3.5), it holds that $x^{k} \rightarrow x^{*}, k \rightarrow \infty$. Thereby, it holds that

$$
\begin{gathered}
\lambda_{j_{0}}^{k} \leq g_{j_{0}}\left(x^{k}\right), \quad \lambda_{j_{0}}^{k} \longrightarrow \lambda_{j_{0}}^{*}<0, \\
g_{j_{0}}\left(x^{k}\right) \longrightarrow g_{j_{0}}\left(x^{*}\right)=0 .
\end{gathered}
$$

While, from (15), there exists some $k_{0}$, such that, for $k \geq k_{0}$,

$$
g_{j_{0}}\left(x^{k}\right) \leq g_{j_{0}}\left(x^{k-1}\right) \leq \cdots \leq g_{j_{0}}\left(x^{k_{0}+1}\right) \leq g_{j_{0}}\left(x^{k_{0}}\right)<0,
$$

it is in contradiction to (46), which shows that $x^{*}$ is a KKT point of (1).

\section{Concluding Remarks}

In this paper, combined with the idea of parallel variable distribution, we proposed a new interior point SSLE algorithm for solving constrained optimization problems. The presented algorithm is a special structure of the objective function or constraints with a special structure. Under some mild conditions, the theoretical analysis shows that the convergence of this algorithm can be obtained.

It is noted that there are still some problems worthy of further discussion such as study of the parallel algorithm with inequality and equality constraints.

\section{Conflict of Interests}

The authors have declared that there is no conflict of interests.

\section{Acknowledgments}

The authors would also like to thank the anonymous referees for the careful reading and helpful comments and suggestions that led to an improved version of this paper. This research was supported by the Foundation of Hunan Provincial Education Department under Grant (nos. 12A077, 12C0743, and 13C453) and Scientific Research Fund of Hunan University of Humanities, Science and Technology of China (no. 2012QN04).

\section{References}

[1] P. T. Boggs and J. W. Tolle, "A strategy for global convergence in a sequential quadratic programming algorithm," SIAM Journal on Numerical Analysis, vol. 26, no. 3, pp. 600-623, 1989.

[2] C. T. Lawrence and A. L. Tits, "Nonlinear equality constraints in feasible sequential quadratic programming," Optimization Methods and Software, vol. 6, no. 4, pp. 265-282, 1996. 
[3] Z. Zhu and J. Jian, "An efficient feasible SQP algorithm for inequality constrained optimization," Nonlinear Analysis: Real World Applications, vol. 10, no. 2, pp. 1220-1228, 2009.

[4] Z. Luo, G. Chen, S. Luo et al., "Improved feasible SQP algorithm for nonlinear programs with equality constrained subproblems," Journal of Computers, vol. 8, no. 6, pp. 1496-1503, 2013.

[5] E. R. Panier, A. L. Tits, and J. N. Herskovits, "QP-free, globally convergent, locally superlinearly convergent algorithm for inequality constrained optimization," SIAM Journal on Control and Optimization, vol. 26, no. 4, pp. 788-811, 1988.

[6] H. D. Qi and L. Qi, "A new QP-free, globally convergent, locally superlinearly convergent algorithm for inequality constrained optimization," SIAM Journal on Optimization, vol. 11, no. 1, pp. 113-132, 2001.

[7] L. Chen, Y. Wang, and G. He, "A feasible active set QP-free method for nonlinear programming," SIAM Journal on Optimization, vol. 17, no. 2, pp. 401-429, 2006.

[8] Z. Zhu, "An interior point type QP-free algorithm with superlinear convergence for inequality constrained optimization," Applied Mathematical Modelling, vol. 31, no. 6, pp. 1201-1212, 2007.

[9] W. X. Cheng, C. C. Huang, and J. B. Jian, "An improved infeasible SSLE method for constrained optimization without strict complementarity," Computers \& Operations Research, vol. 40, no. 5, pp. 1506-1515, 2013.

[10] M. C. Ferris and O. L. Mangasarian, "Parallel variable distribution," SIAM Journal on Optimization, vol. 4, no. 4, pp. 815-832, 1994.

[11] C. A. Sagastizábal and M. V. Solodov, "Parallel variable distribution for constrained optimization," Computational Optimization and Applications, vol. 22, no. 1, pp. 111-131, 2002.

[12] C. Han, Y. Wang, and G. He, "On the convergence of asynchronous parallel algorithm for large-scale linearly constrained minimization problem," Applied Mathematics and Computation, vol. 211, no. 2, pp. 434-441, 2009.

[13] F. Zheng, C. Han, and Y. Wang, "Parallel SSLE algorithm for large scale constrained optimization," Applied Mathematics and Computation, vol. 217, no. 12, pp. 5377-5384, 2011. 


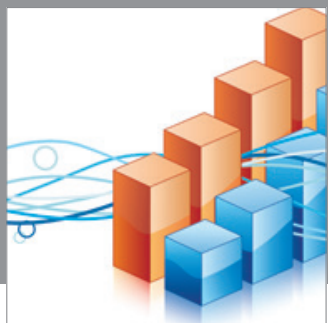

Advances in

Operations Research

mansans

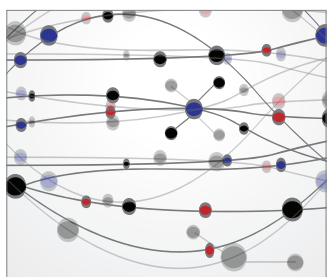

The Scientific World Journal
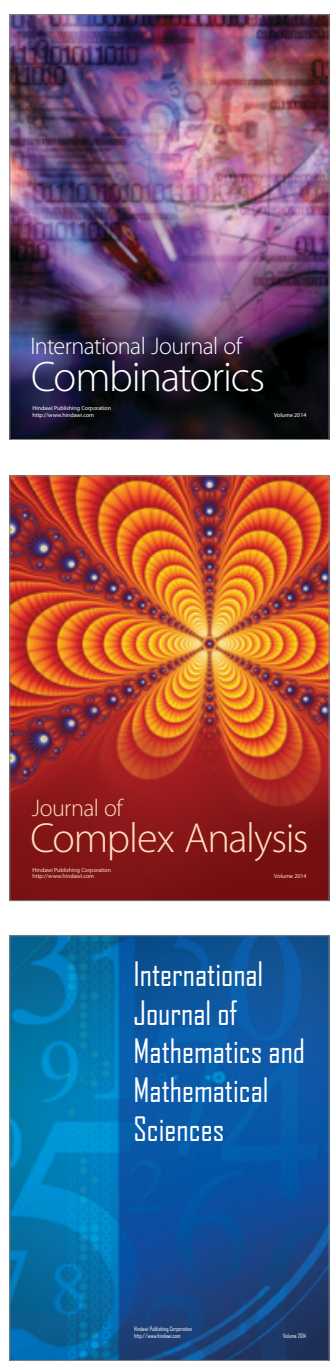
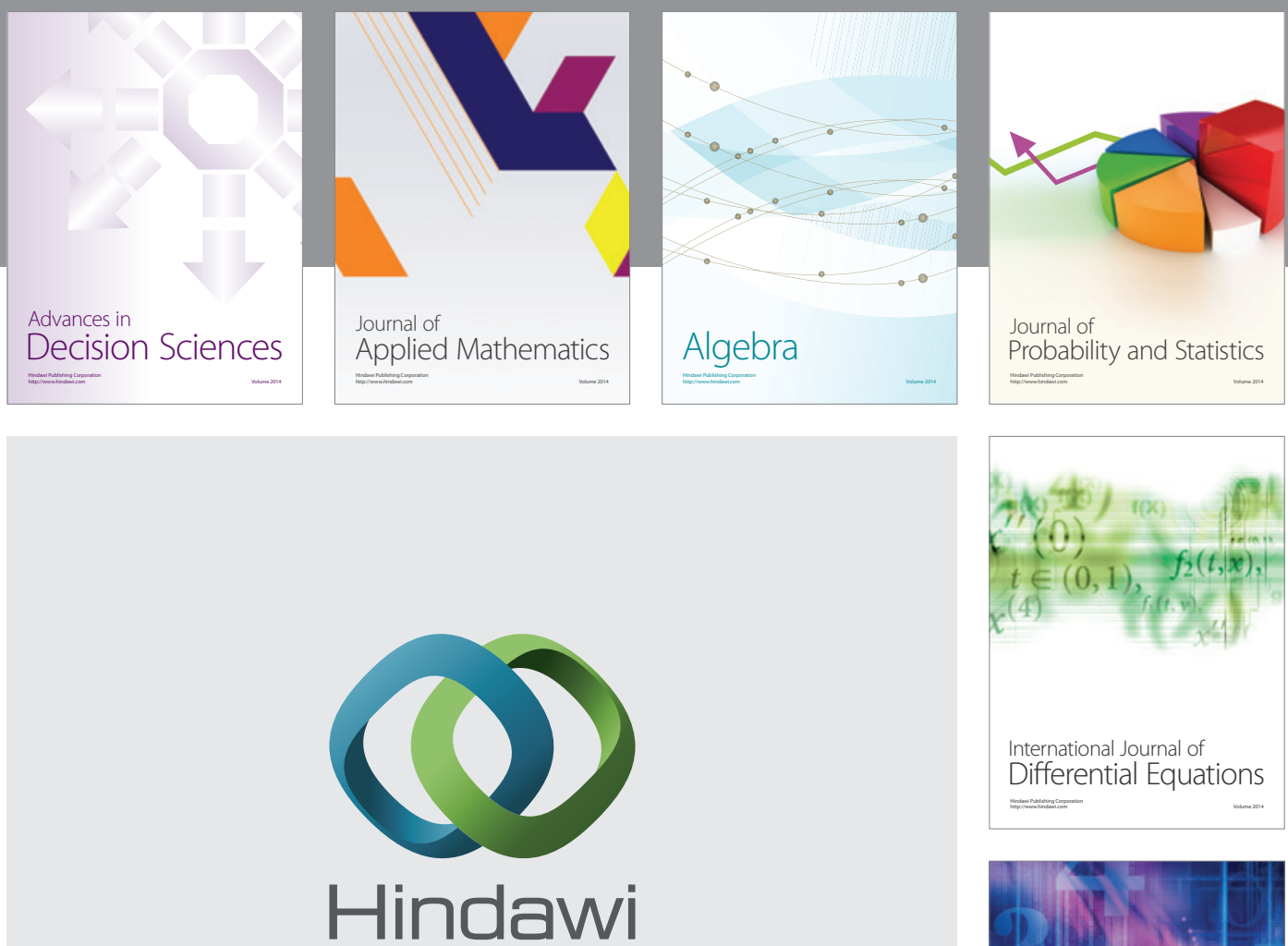

Submit your manuscripts at http://www.hindawi.com
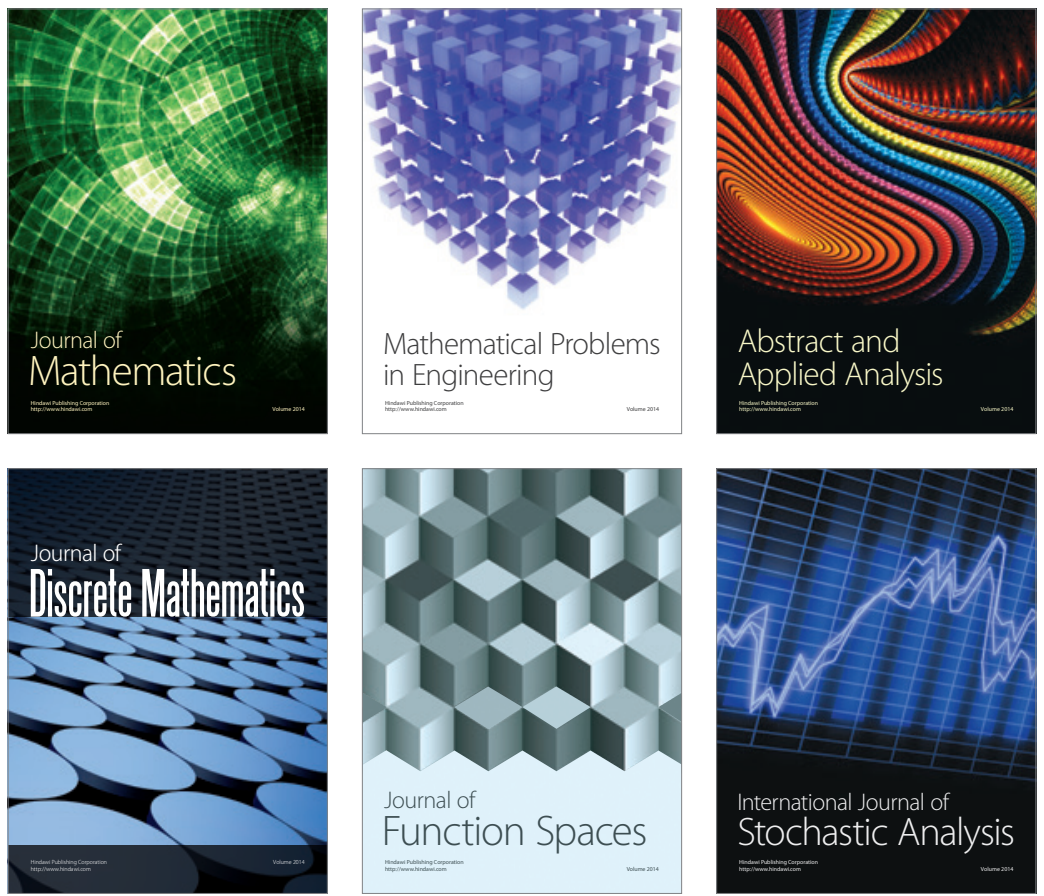

Journal of

Function Spaces

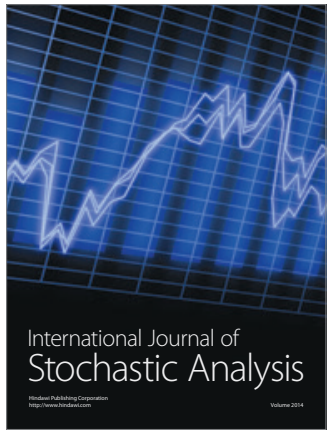

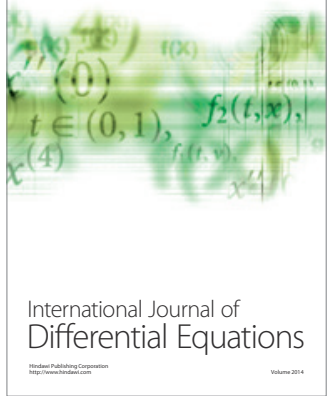
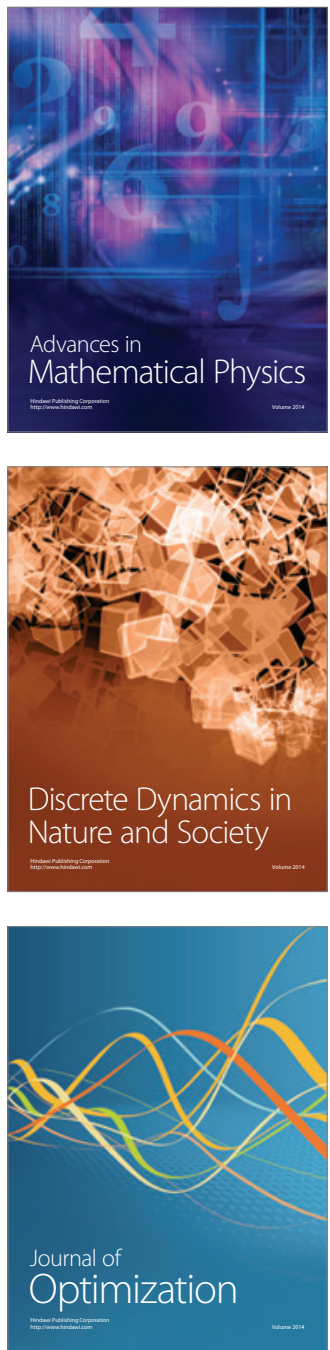